\title{
Sistema Integral para la Gestión del Programa de Tutorías en el Instituto Tecnológico de Tehuacán
}

\section{Integral System for the Management of the Tutorials Program at the Technological Institute of Tehuacán}

\author{
GARCÍA-ORTEGA, Irene†*, VÁSQUEZ-GAMBOA, Saira Antonieta, RODRÍGUEZ-RAMÍREZ, \\ Felipe y SANDOVAL-GONZÁLEZ, Yeyetzin
}

Instituto Tecnológico de Tehuacán / Tecnológico Nacional de México

ID 1 Autor: Irene, García-Ortega / ORC ID: 0000-0001-9638-7577, CVU CONACYT ID: 997104

ID 1 ${ }^{\text {er }}$ Coautor: Saira Antonieta, Vásquez-Gamboa / ORC ID: 0000-0001-7031-0467, CVU CONACYT ID: 997110

ID $2^{\text {do }}$ Coautor: Felipe, Rodríguez-Ramírez / ORC ID: 0000-0002-1991-3390, CVU CONACYT ID: 899136

ID $3^{\text {er }}$ Coautor: Yeyetzin, Sandoval-González / ORC ID: 0000-0002-0963-8540, CVU CONACYT ID: 608569

DOI: $10.35429 / J T A E .2019 .8 .3 .27 .40$

Recibido: 30 de Abril, 2019; Aceptado 30 de Junio 2019

Resumen

La tutoría en los Institutos Tecnológicos ha sido implementada como una estrategia de apoyo para la formación integral del alumno, donde el docente se convierte en acompañante en su proceso de formación profesional, desarrollando actividades que tienen el propósito de estimular el desarrollo humano integral del estudiante. El presente trabajo presenta el diseño de un sistema web, para apoyar la gestión del programa de tutorías, y el quehacer tutorial, involucra a cada uno de los diferentes actores (coordinador Institucional, coordinador de tutorías del área, jefe de departamento, tutorados y tutores). Este sistema web fue el resultado de la investigación Análisis de las tutorías en el Instituto Tecnológico de Tehuacán cuyo propósito fue conocer desde la experiencia de los actores el programa para proponer una estrategia que favoreciera la tutoría. La investigación se desarrolló utilizando una metodología cualitativa, donde se obtiene información utilizando instrumentos como la observación, entrevistas y encuestas. Con este sistema se busca favorecer el programa de tutorías en el logro de sus objetivos y en consecuencia en el logro de las metas institucionales del Instituto Tecnológico de Tehuacán.

Tutoría, Programa de Tutoría, Acción tutorial

\begin{abstract}
The tutoring in the Technological Institutes has been implemented as a support strategy for the integral formation of the student, where the teacher becomes his companion in his professional training process, developing activities that have the purpose of stimulating the integral human development of the student. The present work presents the design of a web system, to support the management of the tutorial program, and the tutorial task, involves each of the different actors (Institutional coordinator, coordinator of area tutorials, department head, tutors and tutors). This web system was the result of the investigation Analysis of the tutorials at the Technological Institute of Tehuacán whose purpose was to learn from the experience of the actors the program to propose a strategy that would favor tutoring. The research was developed using a qualitative methodology, where information is obtained using instruments such as observation, interviews and surveys. This system seeks to favor the mentoring program in the achievement of its objectives and consequently in the achievement of the institutional goals of the Technological Institute of Tehuacán.
\end{abstract}

Tutoring, Tutoring Program, Tutorial action

Citación: GARCÍA-ORTEGA, Irene, VÁSQUEZ-GAMBOA, Saira Antonieta, RODRÍGUEZ-RAMÍREZ, Felipe y SANDOVAL-GONZÁLEZ, Yeyetzin. Sistema Integral para la Gestión del Programa de Tutorías en el Instituto Tecnológico de Tehuacán. Revista de Tecnología y Educación. 2019. 3-8: 27-40

\footnotetext{
* Correspondencia del Autor (Correo electrónico: irenegarcia@ittehuacan.edu.mx)

$\dagger$ Investigador contribuyendo como primer autor.
} 


\section{Introducción}

La tutoría es una herramienta institucional orientada al mejoramiento de la calidad de la educación en nivel superior, y una alternativa para contribuir a abatir los problemas de deserción, reprobación y los bajos índices de eficiencia terminal (ANUIES, sf). Dada la importancia que tiene "se han desarrollado diversas investigaciones donde se muestra la necesidad de orientar a los estudiantes de nivel superior en diferentes contextos, y donde se han apoyado y fomentado propuestas de acciones a desarrollar encaminadas a la orientación, asesoramiento y tutela de los estudiantes" (García , 2018).

En investigaciones realizadas, se ha mencionado como el avance tecnológico y "la implementación de las Tecnologías de la Información y Comunicación (TIC) desde el nuevo paradigma formativo socioconstructivista, el cual está centrado en el estudiante, exige el desarrollo de nuevas metodologías docentes" (Rodríguez, García, \& Vásquez , 2016). En otras investigaciones se menciona también "el uso de herramientas tecnológicas en la acción tutorial mejora la función docente, permitiendo abarcar mayor número de situaciones con posibilidades de éxito, ofreciendo una mayor individualización, y aumentando la eficiencia en el trabajo" (Torres, 2013, pág. 32).

En el Instituto Tecnológico de Tehuacán se desarrolló una investigación, para analizar el programa de tutorías en la carrera de Ingeniería en Sistemas Computacionales, cómo se realizaba la atención a los alumnos tutorados, cómo se llevaba a cabo la atención de problemáticas, la programación de actividades de formación integral, y los efectos en los objetivos del programa de tutorías. Como resultado de esta investigación se propone como una estrategia, la implementación de un sistema que apoye a la tutoría.

Con el desarrollo del sistema se busca sistematizar las actividades que se desarrollan para apoyar la gestión del programa de tutorías y el quehacer tutorial, e involucrar en él a cada uno de los diferentes actores (coordinador Institucional, coordinador de área, jefe de departamento, tutorados y tutores).
Los actores del programa de tutorías tienen un perfil asignado y un espacio para poder registrar, compartir y consultar información. Pueden crear actividades programadas en el PIT (Programa Institucional de Tutorías), llevando un control tanto de las asistencias a sesiones grupales e individuales, así como a cada una de las actividades de formación integral, además de contar con una herramienta de comunicación entre cada uno de los actores de manera permanente, favoreciendo de esta manera el programa de tutorías para que se desarrolle de una manera más efectiva y eficiente y logre así tener un impacto en los índices de deserción y reprobación ya que hasta la fecha aún no se ha logrado.

Es importante la investigación ya que de esta manera se pueden proponer estrategias en beneficio de las tutorías en el Instituto Tecnológico de Tehuacán, con esto beneficiar en primera instancia a los alumnos ya que se les brindará un apoyo más efectivo.

Beneficia también al Instituto Tecnológico de Tehuacán, en el logro de metas institucionales.

Finalmente servirá para otros Institutos Tecnológicos del Tecnológico Nacional de México y de esta manera contribuir con nuestra sociedad al insertar jóvenes exitosos.

Este artículo está integrado de las siguientes secciones:

1. Planteamiento del problema: en este apartado se menciona, el planteamiento del problema que dio pie a la realización de la investigación, el objetivo general, objetivos específicos y pregunta de investigación.

2. Metodología: sección que muestra el desarrollo del proyecto y la metodología utilizada.

3. Desarrollo: En este apartado se presenta el sistema web, como propuesta de apoyo al programa de tutorías.

4. Resultados: en esta sección se presentan los resultados de la implementación del sistema web y propuestas de mejora. 
5. Conclusiones: en este espacio se encuentran las conclusiones del trabajo.

6. Sugerencias: en este apartado se muestran algunas sugerencias.

7. Agradecimientos: en este apartado se presentan los agradecimientos a las diferentes instancias y personas que apoyaron la realización del proyecto de investigación.

8. Referencias bibliográficas: en esta sección se presentan las referencias bibliográficas en las que se apoyó esta investigación.

\section{Planteamiento del problema}

La tutoría ha sido implementada en las carreras del Instituto Tecnológico de Tehuacán desde el año 2006, a través de la acción tutorial que realiza el tutor se brinda atención y acompañamiento a los estudiantes de primero y segundo semestre, ya sea de manera grupal o individual con la finalidad de que durante sus estudios logren un desempeño adecuado y en consecuencia favorecer a disminuir el rezago, la reprobación y la deserción, sin embargo, la tutoría no ha sido eficiente para disminuir estos índices.

En el Instituto Tecnológico de Tehuacán se llevó a cabo una investigación en el año 2016-2017, tomando como campo de estudio la carrera de Ingeniería en Sistemas Computacionales, para evaluar la situación y poder intervenir y proponer estrategias en favor de la tutoría y sus objetivos.

En el Gráfico 1, se presenta un historial de los índices de reprobación de cada una de las materias correspondientes a los alumnos primer semestre de la carrera de Ingeniería en Sistemas Computacionales, periodo enero-junio/2014 a enero-junio/2016 (información proporcionada por el Departamento de Servicios Escolares).

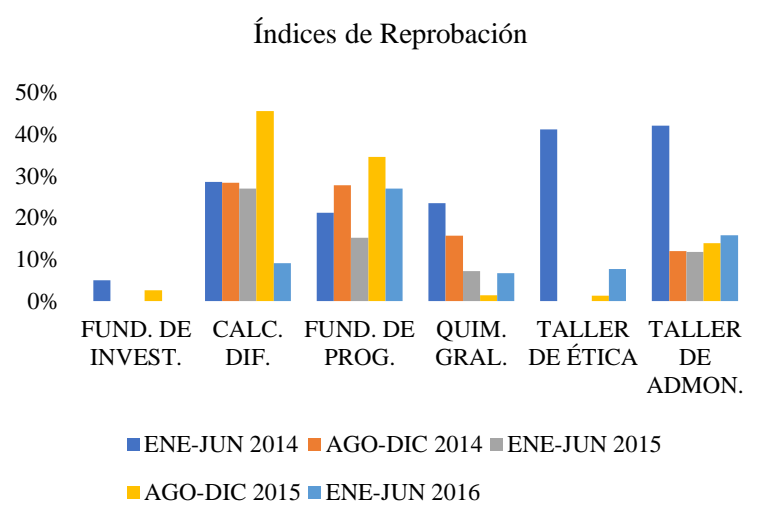

Gráfico 1 Índices de reprobación periodos enero-jun 2014- enero junio 2016

Fuente: (García, 2018)

En este gráfico se observa cómo se presentaron en cada uno de los periodos altos índices de reprobación. Por otro lado, en relación a los alumnos que desertan, en el Gráfico 2 se muestra un historial de los índices de deserción de los alumnos de primer semestre, desde el periodo enero-junio 2014 a enero-junio 2016.

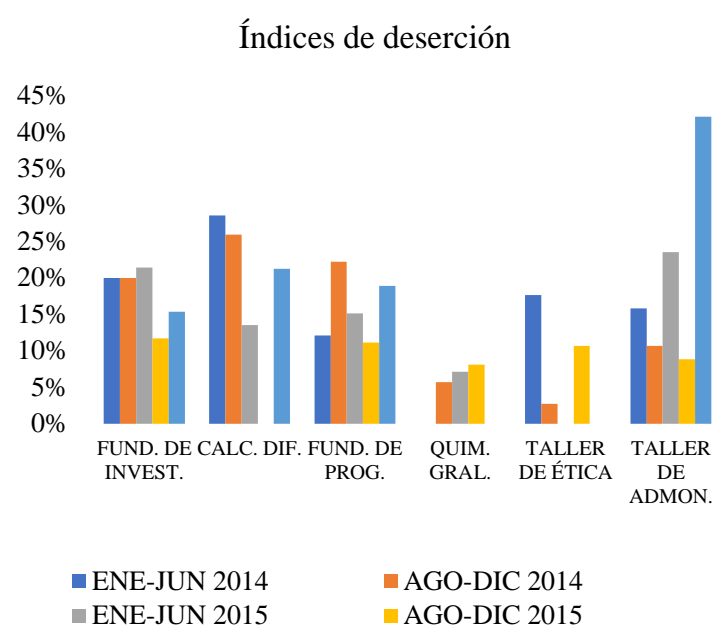

Gráfico 2 Índices de deserción en los periodos enerojunio 2014- enero junio 2016

Fuente: (García , 2018)

En este gráfico se observa cómo se presentaron en cada periodo altos índices de deserción. Es importante mencionar que en primer semestre es donde se dan los más altos índices de reprobación y deserción en la carrera.

Ante los altos índices que se seguían presentando y la existencia de la tutoría que no los favorece, se realiza una investigación y se propone la implementación de un sistema web que apoye la acción tutorial en la carrera de Ingeniería en Sistemas Computacionales. 
Con la investigación realizada se obtuvieron resultados satisfactorios en el área, ya que se atendieron aspectos para favorecer la acción tutorial en beneficio de la tutoría y se dio a conocer a los directivos los beneficios que se obtuvieron, en enero-junio 2017 del grupo de nuevo ingreso se atendieron problemáticas en tiempo, se canalizaron a apoyo psicológico a 2 alumnos en riesgo de deserción, se brindaron asesorías académicas a 4 alumnos de nuevo ingreso y al finalizar el semestre solo hubieron 2 alumnos desertores que no asistieron desde el primer día, los índices de reprobación disminuyeron también ya que de 13 alumnos que ingresaron 2 reprobaron debido a que no se presentaron desde el inicio.

Cabe mencionar que el ingreso de alumnos para el periodo agosto-diciembre 2017 fue mayor y en este momento los tutores muestran dificultad y se les complica dar una eficiente atención tutorial a grupos más grandes. El trabajo desde la elaboración de diagnósticos se hace más complejo, una de las razones por la cantidad de instrumentos de diagnóstico inicial que se deben aplicar y posteriormente evaluar, además sigue presente el descontrol que existe en relación a la solicitud de atención de necesidades, a la canalización de alumnos, a las actividades de formación integral y académica. Esta información se obtuvo después de aplicar instrumentos a los actores del programa, para conocer la situación en cada una de las áreas académicas y en general la situación de la tutoría en el Instituto Tecnológico de Tehuacán.

Se dio a conocer esta información a directivos y se presenta la propuesta para desarrollar el proyecto y se autoriza desarrollar un sistema web que integre la acción tutorial que automatice actividades importantes para la gestión del programa desde los diagnósticos iniciales y la gestión de las actividades de formación integral y académica, de esta manera contar un sistema integral de tutorías que permita automatizar la acción tutorial y poder tener información importante que permita tomar decisiones y acciones, sobre todo que permita contar con información a partir de la cual se genere el PIT, y así ir generando una cultura de las tutorías, que realmente impacte en favor de sus objetivos y en el cumplimiento de las metas institucionales.
En la Figura 1 se muestra la presentación del proyecto a los directivos.

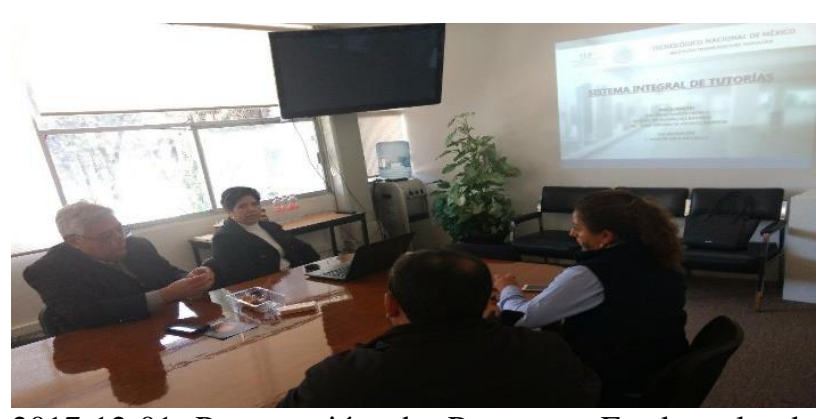

2017-12-01 Presentación de Proyecto. En la sala de juntas de la dirección se llevó a cabo la presentación del proyecto "Sistema Integral de Tutorías", al M.A.C. Rodolfo Medrano Castillo, Subdirector Académico de nuestro Instituto, este proyecto fue presentado por los docentes de la carrera de Ingeniería en Sistemas Computacionales. M.C. Irene García Ortega, M.E. Saira Antonieta Vásquez Gamboa y el M.T.I. Felipe Rodríguez Ramírez

Figura 1 Presentación del proyecto de tutorías Fuente:

(https://www.facebook.com/institutotecnologicotehuacan. itteh/)

\section{Objetivo General:}

Implementar un Sistema Integral web para la gestión del programa de tutorías en el Instituto Tecnológico de Tehuacán y favorecer los objetivos del programa de Tutorías.

Una vez que se desarrolló la investigación en relación a la falta de eficiencia de las tutorías en la carrera de Ingeniería en Sistemas en el Instituto Tecnológico de Tehuacán, se propone el desarrollo de un Sistema web que integre a cada una de las áreas académicas para que apoye en el desarrollo de la acción tutorial. En este artículo se presentan los resultados del desarrollo del proyecto y su implementación al integrar a todos los actores del programa de tutorías.

\section{Pregunta de investigación:}

¿Implementar un sistema web que automatice las acciones de los actores favorecerá al programa de tutorías en el Instituto Tecnológico de Tehuacán? 


\section{Metodología}

La investigación se desarrolló bajo una metodología de tipo cualitativa la cual "se interesa por captar la realidad social a través de los ojos de la gente que está siendo estudiada" (Monje, 2011). El método que se utilizó fue de investigación-acción. el cual puede llevarse a cabo por personas o grupos que llevan a cabo una actividad colectiva en bien de todos, con miras a establecer cambios apropiados en la situación estudiada (Colmenares E. \& Piñeiro M., 2008). Este método sigue un modelo en espiral en ciclos sucesivos, compuestos por las fases de planificar, actuar, observar y reflexionar.

Para la investigación se proponen 3 ciclos, el primero tiene como objetivo establecer una propuesta de mejora, que fue el objetivo del trabajo de investigación previo que se realizó, donde el resultado fue establecer como propuesta de mejora el desarrollo e implementación de un sistema web que automatizará las acciones de los actores del programa de tutorías en el Instituto Tecnológico de Tehuacán. El segundo ciclo tiene como finalidad implementar el sistema y evaluar los resultados de su implementación, que es el fin de esta investigación y un tercer ciclo tendrá como objetivo evaluar el impacto del sistema web en los objetivos del programa de tutorías en el Instituto Tecnológico de Tehuacán.

En este trabajo el estudio se basó en observar a los diversos actores del programa de tutorías, para conocer a partir de su propia experiencia como llevan a cabo la acción tutorial, para comprender que actividades desempeña cada uno y automatizarlas e integrarlas en un sistema web que sirva de apoyo para cada uno de ellos con la finalidad de favorecer los objetivos de la tutoría.

En la investigación se aplicaron diversas técnicas como son entrevistas a los diferentes actores: coordinadora institucional de tutorías, coordinadores de tutorías de las áreas académicas, tutores, jefes de departamento y tutorados, también se aplicó como técnica de investigación la observación y encuestas a alumnos tutorados.

Los pasos a seguir en el desarrollo de este proyecto fueron:
Aplicar los instrumentos de investigación para conocer las necesidades desde el perfil de cada uno de los actores.

Analizar la información recolectada.

Desarrollar un diagrama donde se represente el proceso en general desde que inicia el programa hasta que culmina, donde se incluya a cada uno de los actores para entender cuál es su participación y en qué momento debe realizarla, así como la importancia de llevar en tiempo y forma su participación.

Modelar los procesos que lleva cabo cada uno de los actores.

\section{Implementar el Sistema.}

Capacitar a los diferentes usuarios del sistema, participando en talleres donde se explica el proceso general, utilizando el diagrama propuesto, y el perfil correspondiente en el sistema, donde se muestre dónde y cuándo utilizarlo para el desarrollo de sus actividades.

Al final del semestre entrevistar nuevamente a los actores para conocer su experiencia en el uso del sistema en favor de su función dentro del programa de tutorías.

\section{Desarrollo}

Para aplicar los instrumentos y analizar la información, se solicitó autorización para poder aplicar diversos instrumentos, dando inicio con diferentes entrevistas realizadas a la coordinadora institucional de tutorías, jefes de departamento, coordinadores de tutorías de las diferentes áreas académicas, tutores y tutorados.

Se asistió como observador a diferentes reuniones de tutoría, dando inicio con la reunión del coordinador institucional de tutorías con los diferentes tutores de las áreas académicas, posteriormente se asistió a juntas de tutores con la coordinadora de tutorías del área y sesiones grupales, para conocer aspectos relacionados con la gestión del programa. A la coordinadora institucional se le entrevistó con preguntas relacionadas con las actividades que desempeña, entre estas acerca del programa institucional de tutorías, y cómo implementa su programa, que problemática se presenta en el proceso de implementación y si realiza un plan de mejora continua. 
También se participó como observador en las reuniones de inicio al programa donde se convocaba a los coordinadores de tutorías de las áreas académicas, para conocer el número de personas que asistían, que preguntas realizaban en relación al Programa Institucional que se les presentaba y que problemáticas exponían.

Se entrevistaron a 42 tutores de los 51 registrados, en la mayoría de los casos fueron sesiones por grupos de tutores, donde daban a conocer como se realizaba la acción tutorial, los problemas que tenían, la entrevistas se realizaron con un guion de 10 preguntas abiertas donde el objetivo principal era conocer como llevaban a cabo su acción tutorial, que problemas enfrentaban en este proceso, y también se les aplicó una encuesta de 10 preguntas cerradas donde el objetivo estaba en detectar a partir de las respuestas su experiencia y conocimiento para llevar a cabo la acción tutorial. En estas reuniones también se observaba junto con la presencia de otros 2 colaboradores aspectos de valor a considerar en la investigación.

Se aplicaron encuestas abiertas a grupos de alumnos tutorados de primero y de segundo semestre de las diferentes áreas para conocer como participan en el programa, que información necesitaban conocer, que necesidades de atención requerían, como se les había atendido, si la atención había sido oportuna, de qué manera se les podía facilitar la comunicación con sus tutores.

Se encuestaron a 352 estudiantes tutorados de primero y segundo semestre, la encuesta estaba compuesta de 20 items, de los cuales 2 eran preguntas abiertas, tres de escala de Likert con ítems relacionados con la relación con el tutor, cumplimiento del tutor y actitud del tutor, y 15 preguntas cerradas relacionadas con las sesiones de tutorías, la efectividad y confianza en el programa de tutorías.

Como resultado de la aplicación de los instrumentos y del análisis de la información se obtuvo lo siguiente:

Es necesario que exista una vista o un perfil para cada uno de los actores del programa donde encuentre opciones para desarrollar las actividades que le corresponden.
Entre estas actividades se encuentran los siguientes aspectos que son importante considerarlos ya que son indicadores que resultaron de la aplicación de los instrumentos de investigación.

Por falta de capacitación se les dificulta la elaboración de sus Planes de Acción Tutorial, y no saben qué actividades realizar en sus sesiones grupales. En este caso se presenta el desarrollo de un módulo donde se puede registrar por parte de los coordinadores de las áreas académicas una plantilla donde registre una guía de las posibles actividades que el tutor puede realizar, dependiendo del semestre de sus alumnos tutorados, de esta forma no serán actividades improvisadas.

A los tutores se les dificulta la elaboración de diagnósticos iniciales como resultado de la aplicación de los diferentes instrumentos, se encontró que no se realizan diagnósticos, esto principalmente porque se les hace complicado evaluar los test que se proporcionan como instrumentos de diagnóstico en el manual del tutor. Por lo cual se analizaron cada uno de estos instrumentos y se automatizaron para que en el sistema se registrará la información por parte de los alumnos y el tutor consultara en su perfil los resultados para realizar sus diagnósticos y detección de necesidades para solicitarlas y canalizar a los alumnos si fuera necesario.

Las actividades de formación integral que son programadas tampoco responden a un diagnóstico y se realizan sin llevar un control de las mismas ni de la asistencia y participación de los alumnos por lo cual el impacto en los alumnos tutorados es casi nulo, el alumno pierde interés porque en muchas ocasiones la cantidad de participantes rebasa la capacidad de los espacios, o porque se les invita a asistir cuando la conferencia ya está en curso. Para estas gestiones se automatizan módulos relacionados en los perfiles del tutor, coordinador de tutorías y coordinador institucional.

El reporte final del tutor se elabora de manera manual y refleja lo que ellos finalmente creen que se realizó sin que exista ninguna evidencia de lo que se informa. 
En el perfil tutor del sistema, se podrá llevar un registro cada vez que realice una sesión grupal o individual, de las solicitudes de atención tutorial, de las canalizaciones y de las actividades de formación integral de tal forma que al finalizar el programa el tutor desde el sistema pueda imprimir su reporte donde hay registro de cada una de estas actividades y de la participación y asistencia de sus tutorados.

Los coordinadores de las diferentes áreas académicas no tienen información ni conocimiento de las sesiones grupales que se desarrollan por parte de los tutores. Para desarrollar su informe final de área dependen de la información que les proporciona cada uno de los tutores.

La coordinadora institucional informa vía un oficio o vía telefónica de las diferentes actividades que se programan, muchas de las veces minutos antes de que se lleven a cabo.

El registro de la asistencia a las actividades de formación integral se realiza de manera manual en hojas, que se archivan y no proporcionan ninguna información a las áreas académicas o a los tutores que les permita llevar un control de la participación de sus alumnos y poder darles seguimiento.

La propuesta consiste en desarrollar un sistema web que apoye a la gestión del programa de tutorías. Un sistema web es una aplicación de software que puede utilizarse accediendo a un servidor web a través de internet $o$ de una intranet mediante un navegador (aerus, 2016).

Para el desarrollo del sistema se utilizó la metodología ágil XP la cual promueve el trabajo en equipo, se basa en la retroalimentación continua entre el cliente y el equipo de desarrollo, comunicación fluida entre los participantes (Rizzo \& Córdova, 2015).

Se desarrolló el diagrama del proceso de la tutoría, con la finalidad de que cada uno de los actores vea a la tutoría como un proceso que tiene un objetivo y actividades que realizar, y deje de practicarse como un conjunto de actividades aisladas e improvisadas. Se modelaron los procesos de cada uno de los actores.
Se codificaron cada uno de los perfiles utilizando una arquitectura MVC (Modelo Vista Controlador), este modelo específica que una aplicación consta de un modelo de datos, de información de presentación y de información de control (Alvarez, 2014). Este modelo es utilizado en la implementación de sistemas que requieren del uso de interfaces de usuario, como el que se requiere para la gestión del programa de tutorías.

Se realizaron pruebas de su funcionamiento y en el semestre enero-junio 2019, se implementó en cada una de las áreas académicas del Instituto Tecnológico de Tehuacán.

\section{Resultados}

El desarrollo del sistema se realizó por roles (perfiles) que corresponden a cada uno de los actores del programa de tutorías. Por ser un sistema que integra diferentes vistas, se le agregaron roles necesarios para su funcionamiento, de tal forma que en el sistema se tienen considerados los siguientes roles: Tutorado, tutor, coordinador de tutorías del área académica, jefe de departamento, coordinador institucional y administrador como se muestra en la Figura 2.

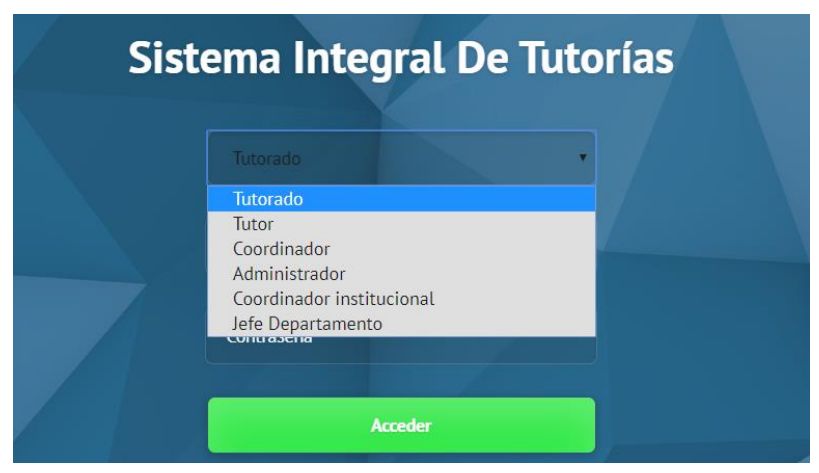

Figura 2 Perfiles que pueden accesar al sistema Fuente (http://tutoriasfinalsistema.itectehuacan.edu.mx/)

Perfil Tutorado: este perfil, corresponde al espacio al que van a ingresar los alumnos que tengan asignada la tutoría. En este espacio tienen acceso a un manual de usuario del sistema, al cuaderno de trabajo de tutoría del estudiante del SNIT (TecNM, Cuaderno de trabajo de tutoría del estudiante, 2013), y de acuerdo al análisis realizado se integran las actividades en las que debe participar el alumno tutorado como parte del programa de tutorías, las cuales quedan integradas como se muestra en la Figura 3. 


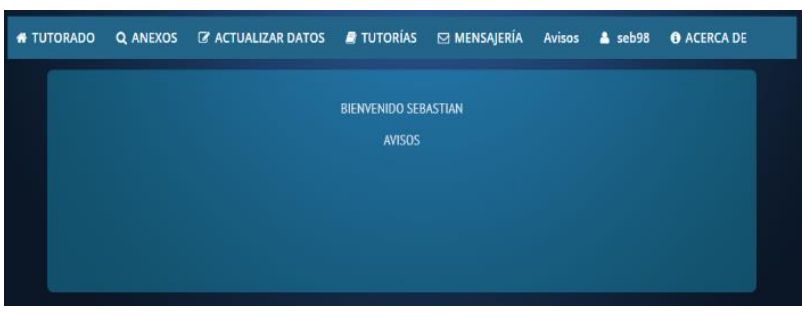

Figura 3 Módulo del Perfil Tutorado Fuente

(http://tutoriasfinalsistema.itectehuacan.edu.mx/vistas/)

Perfil Tutor: corresponde al que van a accesar todos los docentes de las áreas académicas que va a llevar a cabo la tutoría. En su perfil tienen acceso al manual del tutor, manual de usuario del sistema, y de acuerdo al análisis realizado se integran las actividades en las que debe participar dentro del programa de tutorías, las cuales quedan integradas como se muestra en la Figura 4.

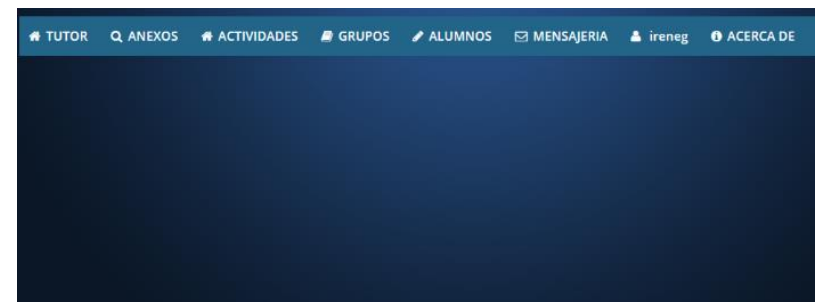

Figura 4 Módulo del perfil tutor

Fuente

(http://tutoriasfinalsistema.itectehuacan.edu.mx/vistas/ID ocente.php)

Perfil coordinador de tutorías del área académica: este perfil tiene acceso a la información de los grupos del área académica a la que corresponde, a los tutores asignados, gestionar las necesidades que los tutores le envíen para ser atendidas por el área académica o para canalizarlas a la coordinadora.

Cada coordinador de las áreas académicas, puede monitorear las sesiones grupales e individuales que se están realizando $\mathrm{y}$ al final del semestre recibir el reporte de tutorías de cada uno de los tutores (anexo 19).

Al mismo tiempo que los tutores generan e imprimen su reporte, el coordinador de las áreas académicas puede imprimir su reporte semestral del departamento académico (anexo 20), consultar la evaluación del tutor del Programa Institucional de Tutoría (anexo 16), y analizar los resultados de la rúbrica para evaluar el desempeño del tutor por parte de los alumnos (anexo 17).
Toda esta información es importante porque sirve al coordinador institucional para implementar estrategias de mejora al programa en los siguientes periodos. El módulo del perfil coordinador de tutorías se muestra en la Figura 5.

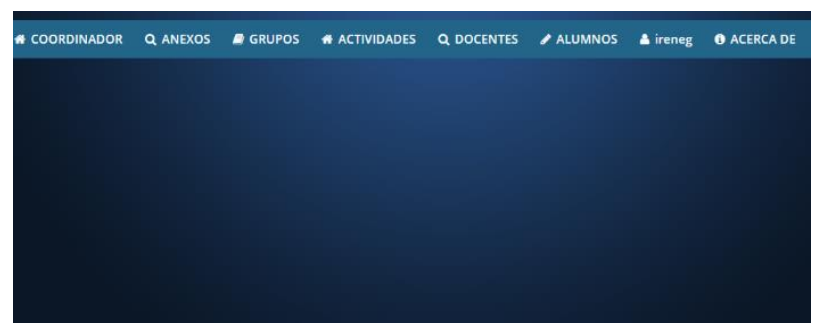

Figura 5 Módulo perfil coordinador de tutoría Fuente (http://tutoriasfinalsistema.itectehuacan.edu.mx/vistas/IC oordinador.php)

Perfil jefe de departamento: en este perfil el jefe de departamento, es el encargado de dar de alta a los docentes tutores y alumnos asignados a cada uno de ellos. Además, se ocupa de la creación de grupos de tutoría del semestre y asignación de tutores y tutorados. También en este perfil puede asignar el rol de coordinador de tutoría del área. La Figura 6, corresponde al perfil del jefe de departamento académico.

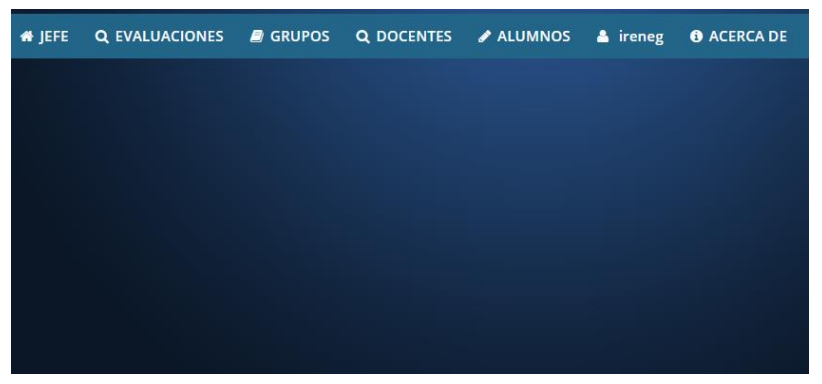

Figura 6 Módulo del perfil Jefe de Departamento

Fuente

(http://tutoriasfinalsistema.itectehuacan.edu.mx/vistas/IJef e.php)

Perfil Coordinador Institucional de tutorías: en este perfil el coordinador institucional, es el encargado de dar inicio al proceso de la tutoría, tiene la responsabilidad de crear y registrar su Plan de Acción Institucional, para que todos los demás actores puedan agregarlo a su Plan de acción y alinear los propios. 
También el coordinador tiene la responsabilidad de crear las primeras actividades de formación integral de acuerdo a su diagnóstico institucional y dar atención a las necesidades ingresadas por los coordinadores de cada una de las áreas académicas, controlar y registrar la asistencia a cada una de las actividades programadas, recibir los reportes finales de cada una de las áreas académicas y generar su reporte semestral.

En la Figura 7 se muestra el módulo del sistema del perfil coordinador institucional.

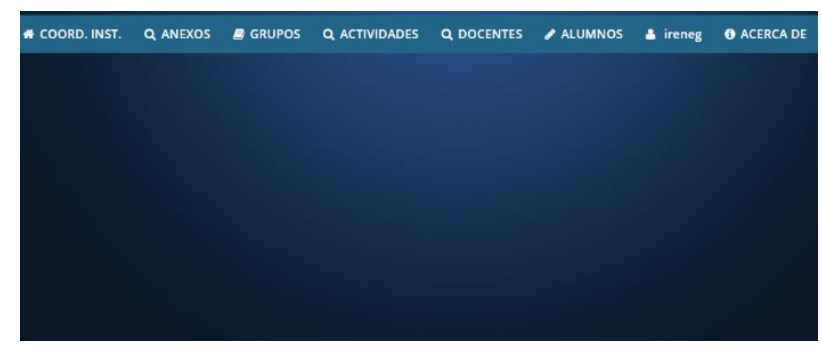

Figura 7 Módulo perfil coordinador institucional

Fuente

(http://tutoriasfinalsistema.itectehuacan.edu.mx/vistas/IC Regional.php)

Perfil administrador: Este perfil cuenta con funciones para ingresar información general para el funcionamiento del sistema, en este perfil se asignan o modifican los roles a los usuarios, se da de alta a usuarios, se puede consultar información en general y recuperar contraseñas para cualquier usuario. En la Figura 8 , se muestra el módulo que corresponde al perfil del administrador.

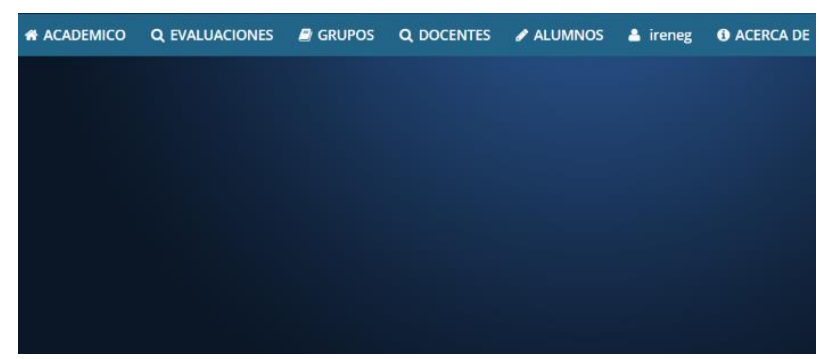

Figura 8 Módulo perfil administrador del sistema Fuente

(http://tutoriasfinalsistema.itectehuacan.edu.mx/vistas/IAd ministrador.php)

Como resultado se tiene un sistema integral web, que puede ser utilizado por cada uno de los actores, y les sirva de apoyo en favor de la tutoría. Una vez concluido el sistema se instala en un servidor para que se pueda acceder por medio de un navegador por parte de los usuarios.
Para poder probarlo de manera integral, se dio un proceso de capacitación para los diferentes usuarios y se proporcionaron manuales de consulta para apoyar el uso del sistema. Se capacitó a cada uno de los jefes de departamento mostrándoles el diagrama del proceso y utilizando el sistema en las opciones que el jefe participa, se les proporcionó un manual de usuario para consulta de sus actividades y del uso del sistema. También se capacitó a los coordinadores de tutorías de las áreas académicas, algunos participaron en la capacitación de jefes y también tuvieron la oportunidad de explorar el sistema y probarlo.

En la Figura 9 se muestra uno de los momentos de capacitación que se proporcionó.

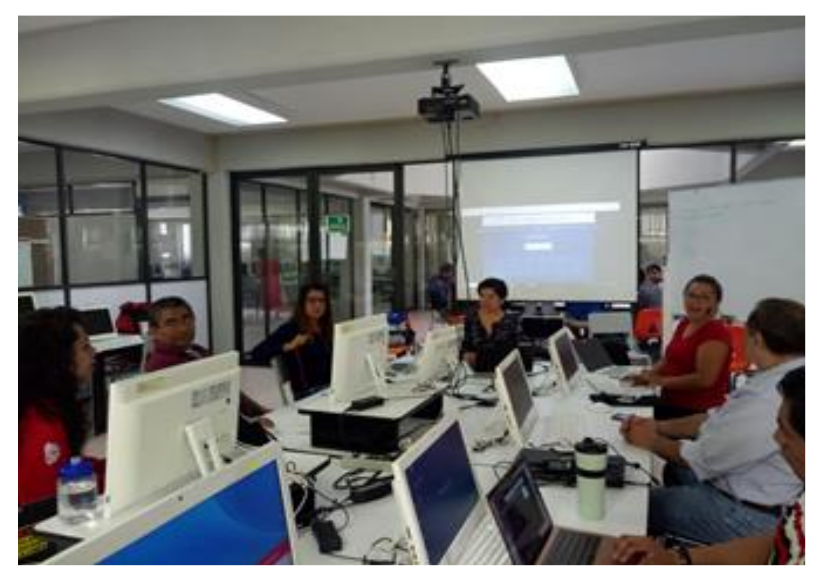

Tehuacán, Puebla a 22 de marzo de 2019. Capacitación a las (os) Coordinadores de tutorías de las diferentes áreas académicas, en la plataforma del Sistema Integral de Tutorías.

Figura 9 Capacitación a Coordinadores de Tutorías Fuente

(https://www.facebook.com/institutotecnologicotehuacan .itteh/)

Se dio capacitación a todos los tutores que en el semestre enero-junio 2019 tenían asignada la tutoría, sin embargo, participaron también docentes de tiempo completo que lo solicitaron porque en algún momento van a ser asignados a la tutoría en los siguientes semestres. Cabe mencionar que los coordinadores de las diferentes áreas académicas participaron también de la capacitación de tutores para conocer el alcance del módulo del tutor y la relación con el módulo del coordinador de área y apoyar a sus tutores en las dudas que se les presenten y dar un uso más eficiente al sistema. En la Figura 10, se muestra la imagen de la capacitación que se proporcionó a los docentes y tutores del departamento de Económico Administrativas. 
En este departamento se incluyen las carreras de Ingeniería en Gestión empresarial, Contador Público y la Licenciatura en Administración.

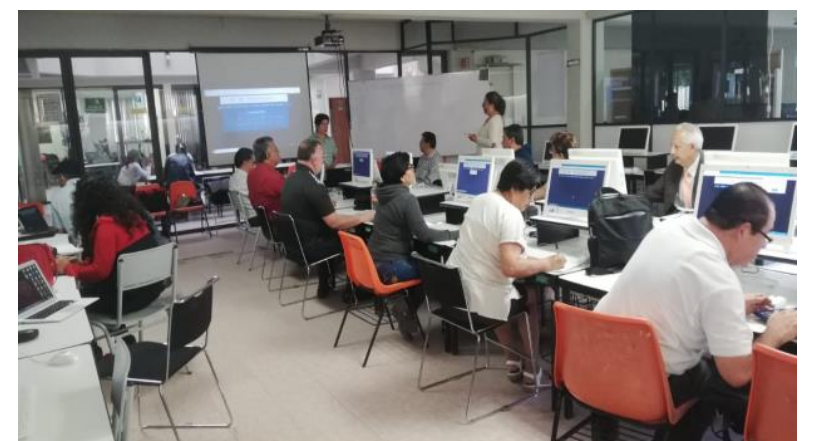

Tehuacán, Puebla a 01 de marzo de 2019. Capacitación de tutores del área del Departamento de Económico Administrativas en el Sistema Integral de Tutorías del TecNM/Instituto Tecnológico de Tehuacán.

Figura 10 Capacitación de tutores del Departamento Económico Administrativas

\section{Fuente:}

https://www.facebook.com/institutotecnologicotehuacan.i tteh/)

En este caso existe un coordinador que administra a las 3 carreras, esto lo permite el sistema si es necesario como en este caso, si no es así y existe un coordinador para cada una de las áreas académicas, cada uno podrá administrar su perfil de manera independiente.

En la Figura 11 se muestra el momento en el que se capacita a los tutores de la carrera de Ingeniería Bioquímica. Docentes de Ing. En Sistemas Computacionales dan capacitación a alumnos de Ing. Civil del primer semestre sobre el uso del software para tutorías.

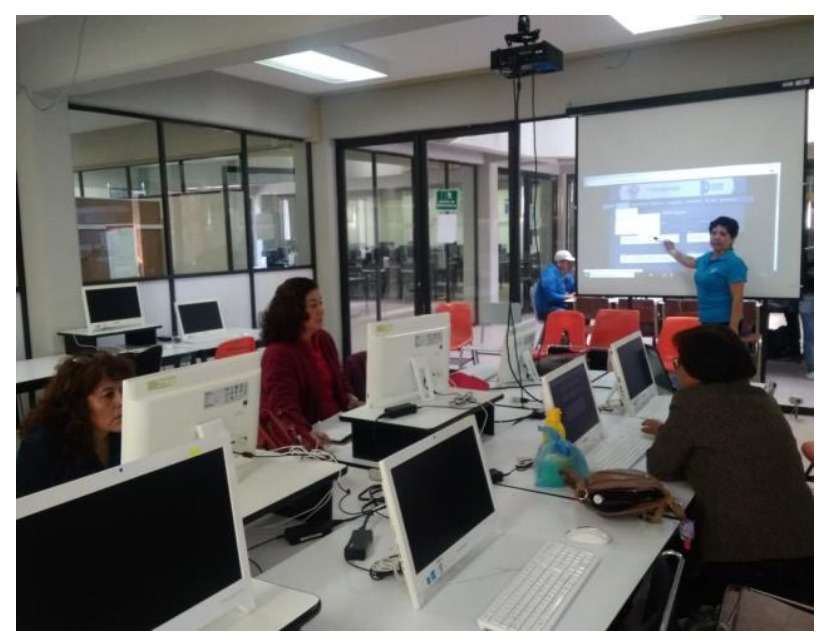

Figura 11 Capacitación a tutores del área de Ing. bioquímica

Fuente: Elaboración Propia
A los alumnos tutorados de manera inicial se les dio una plática donde se les explicó cómo realizar su registro en la plataforma para ser usuarios, se les proporcionó una guía donde se explica paso a paso las actividades que deben realizar para su registro. Este documento está disponible en la página oficial del Instituto Tecnológico de Tehuacán.

Posteriormente en aulas de laboratorio de computo del instituto se les dio una capacitación a alumnos del área de Ing. Bioquímica que así lo solicitaron.

En la Figura 12 se muestra una imagen donde se capacita a un grupo de alumnos de la carrera de Ingeniería Civil.

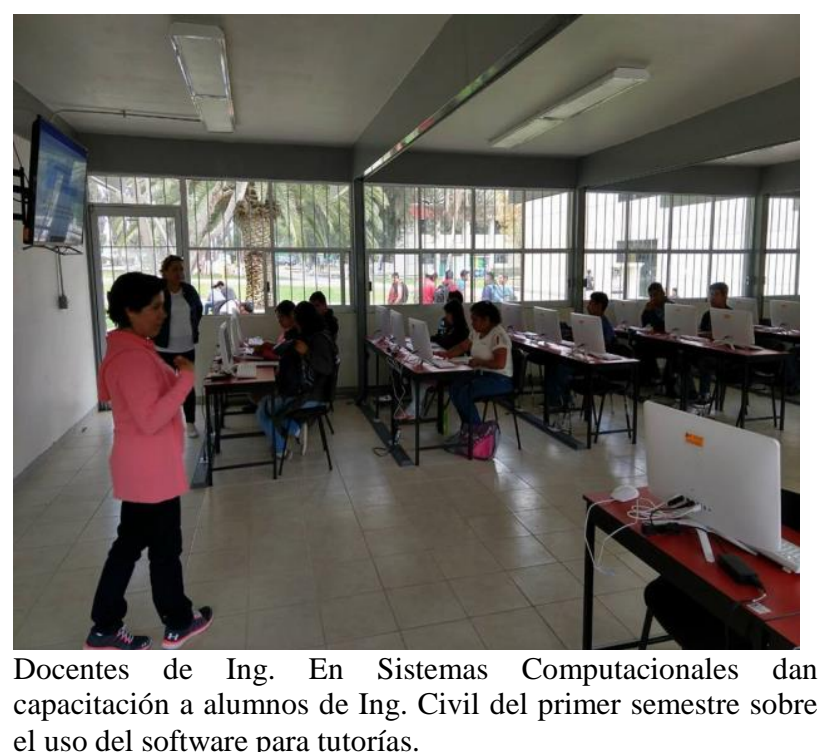

el uso del software para tutorías.

Figura 12 Capacitación a alumnos en el uso del sistema de tutorías

Fuente:

(https://www.facebook.com/institutotecnologicotehuacan. itteh/)

Una vez realizado el proceso de capacitación se procedió a dar inicio con el uso del sistema en cada una de las áreas académicas.

Esta fase es importante porque les permite a los diferentes usuarios el familiarizarse con esta herramienta, y así poder darle un uso más eficiente conforme se vaya utilizando, solo así se podrá evaluar si el sistema impacta y favorece a los índices de reprobación y deserción.

Como resultado de la implementación del sistema se tienen los siguientes datos: 
La cantidad de docentes tutores registrados en el sistema en cada una de las áreas académicas se muestra en la Tabla 1.

\begin{tabular}{|c|c|c|c|}
\hline \multirow[t]{2}{*}{ Área } & \multirow{2}{*}{$\begin{array}{l}\text { No. de } \\
\text { tutores }\end{array}$} & \multicolumn{2}{|c|}{$\begin{array}{l}\text { No. de alumnos } \\
\text { registrados }\end{array}$} \\
\hline & & $1^{\circ}$ & $2^{\circ}$ \\
\hline Ing. Bioquímica & 5 & 32 & 148 \\
\hline Ing. Civil & 2 & 53 & 0 \\
\hline Ing. Gestión & 3 & 0 & 38 \\
\hline Ing. Industrial & 5 & 39 & 179 \\
\hline Ing. Logística & 1 & 0 & 47 \\
\hline Ing. Mecatrónica & 7 & 25 & 155 \\
\hline $\begin{array}{ll}\text { Ingeniería } & \text { en } \\
\text { Sistemas } & \end{array}$ & 9 & 101 & 11 \\
\hline Lic. Administración & 6 & 30 & 71 \\
\hline Contador Público & 8 & 25 & 136 \\
\hline Ing. Electrónica & 7 & 43 & 0 \\
\hline Total & 51 & 318 & 785 \\
\hline
\end{tabular}

Tabla 1 Registro en el sistema de tutores y tutorados Fuente: Elaboración Propia

Como se observa en la Tabla 1 , en el sistema se han registrado al $100 \%$ de alumnos de primero y segundo semestre que son los semestres obligatorios de tutoría para los alumnos en el Tecnológico de Tehuacán, sin embargo, si alguna de las áreas cuenta con los recursos para brindar atención a alumnos de otros semestres lo puede realizar, en particular en el área de Ingeniería electrónica existen alumnos registrados desde primero hasta el doceavo semestre.

\begin{tabular}{|l|r|r|}
\multicolumn{1}{|c}{ Área } & $\begin{array}{c}\text { Tutores } \\
\text { No. docentes } \\
\text { registrados }\end{array}$ & $\begin{array}{c}\text { Cuantos culminaron el } \\
\text { proceso }\end{array}$ \\
\hline Ing. Bioquímica & 5 & 0 \\
\hline Ing. Civil & 2 & 0 \\
\hline Ing. Gestión & 3 & 3 \\
\hline Ing. Industrial & 5 & 2 \\
\hline Ing. Logística & 1 & 0 \\
\hline Ing. Mecatrónica & 7 & 1 \\
\hline Ingeniería en Sistemas & 9 & 9 \\
\hline Lic. Administración & 6 & 6 \\
\hline Contador Público & 8 & 7 \\
\hline Ing. Electrónica & 7 & 0 \\
\hline Total & 53 & 28 \\
\hline
\end{tabular}

Tabla 2 Tutores que concluyeron el proceso Fuente: Elaboración Propia

Con estos resultados de la Tabla 2 se observa que el $53 \%$ de los tutores han concluido el proceso de tutoría, sin embargo, entrevistando a los docentes que no concluyeron su proceso se uno de los factores fue por el momento en el que se inició el proceso ya tenían avanzadas sus actividades y ya no quisieron utilizar el sistema y a otros se les olvido o se les dificultó utilizar el sistema y prefirieron seguir de manera tradicional su acción tutorial.
Algunos docentes de los que, si concluyeron el proceso, manifestaron que no registraron sus sesiones grupales e individuales, así como la solicitud de necesidades para sus tutorados y de actividades de formación integral, esto porque no había realizado sus diagnósticos iniciales o porque olvidaron como hacerlo esto dio lugar a que su reporte final no saliera de manera correcta y nuevamente terminaron por hacerlo de manera manual.

Al entrevistar a estos docentes se observa que realmente sus dudas van más allá del uso del sistema, y lo que se requiere es una capacitación para ser tutores y principalmente de la formación como tutores que se brinda a través del diplomado en formación de tutores donde la finalidad es "ampliar las habilidades del tutor en las dimensiones personal, académica y profesional (TecNM, 2015), porque no solo es el desconocimiento sino también la falta de compromiso que se tiene al ser tutores, así como de asumir la responsabilidad iniciando con la sensibilización que debemos tener para brindar un verdadero apoyo a los estudiantes.

Cabe mencionar que al finalizar las capacitaciones se preguntaba a los diferentes usuarios acerca del sistema, a lo cual el $95 \%$ manifestó que uso estaba sencillo, realizaron diferentes actividades durante la capacitación y no se les dificulto realizarlas, la interfaz es muy amigable y general hubo buena aceptación para utilizar el sistema.

También se entrevistó y apoyo durante todo el semestre a una tutora que a pesar de la edad y de las dificultades que implica el manejo de la tecnología, trabajo de manera muy satisfactoria con el sistema, usándolo desde el inicio hasta el final culminando con la impresión de su reporte final y manifestando que está muy contenta con el sistema ya que le permite tener disponible la información de sus alumnos tutorados, estar en comunicación constante con ellos, llevar un control de su participación en las diferentes actividades que se le asignan y le permite llevar un seguimiento en semestres subsecuentes.

Entrevistando a los alumnos el $90 \%$ de alumnos registrados manifiestan que el sistema es sencillo en su uso y no tuvieron dificultad para contestar sus instrumentos de diagnóstico. 
También se observa como los alumnos asisten de manera regular sin problema a su lugar de sesiones grupales, el $100 \%$ de ellos conocen a su tutor y al preguntarles acerca de si se les ha atendido en sus necesidades de atención se observa que en el área de Ingeniería en Sistemas Computacionales el $100 \%$ de alumnos entrevistados manifestó que si se les ha atendido y se les ha canalizado, es importante mencionar que debido a las necesidades presentadas por diversos tutores, en el Tecnológico de Tehuacán se ha contratado el servicio de 2 psicólogos que están atendiendo específicamente necesidades de alumnos canalizados, esto genera confianza en los estudiantes ya que anteriormente aunque se solicitará este apoyo muchas veces la atención no era la esperada por el estudiante o no se le atendía ya que la psicóloga tenía otras actividades además de la atención de alumnos.

En el área de Sistemas la atención psicológica ha sido importante en el apoyo a alumnos ya que se han presentado casos especiales que requieren ser canalizados y que gracias a su atención se ha dado seguimiento de manera efectiva. Estos alumnos continúan estudiando de manera exitosa la carrera sin optar por la deserción.

Se culmina el proceso con la evaluación del Programa de tutorías y de la acción tutorial, esto es importante ya que sirve como retroalimentación sin embargo debido a que no todos los docentes han culminado el proceso esta actividad no se ha realizado por el $100 \%$ de participantes.

\section{Conclusiones}

Las tutorías son un compromiso muy importante que como docente se adquiere, el tutor es uno de los actores claves, y por lo tanto se necesita que el docente esté capacitado para saber identificar problemas de índole académico, de salud, psicopedagógico del tutorado y poder canalizar a la instancia correspondiente para su atención, todo en el marco de una relación más estrecha que la establecida en un aula durante el semestre (TecNM, 2013).
En esta investigación se encontró que la falta de capacitación es un factor importante que ha propiciado la deficiente acción tutorial por parte de los tutores, así también la falta de un Programa Institucional derivado de un diagnóstico de necesidades, la falta de retroalimentación, también ha sido factor de una acción tutorial improvisada, sin un plan de acción y sin objetivos, por lo cual se ha proporcionado para los tutores plantillas de actividades a partir de la cual los tutores puedan planear las actividades, esto ha dado buenos resultados ya que anteriormente la mayoría de tutores no elaboraban un Plan de Acción Tutorial.

Se desarrolló un sistema que permite proporcionar al tutor información inicial de los alumnos tutorados y con los resultados de la aplicación de test propuestos en el manual del tutor puede conocer el nivel de autoestima de los alumnos, problemas de salud, emocionales, académicos, y realizar un Plan de Acción Tutorial derivado de las necesidades que presentan sus alumnos tutorados, esta información puede estar disponible desde el momento en que inicia el semestre.

El sistema permite llevar un control y registro de las actividades a las que se canaliza un alumno, así como a las actividades de formación integral en las que debe participar y llevar un registro de su asistencia a las mismas.

El sistema ha tenido resultados satisfactorios en el que se han integrado el total de las áreas académicas, donde cada uno de los actores tiene un espacio asignado y actividades a desarrollar dentro del mismo que ayudan a facilitar la acción tutorial.

Hasta el momento ha crecido el interés de los tutores por el uso del sistema, a los estudiantes se les facilita el utilizarlo, y participan con mayor interés en las sesiones de tutorías, el $100 \%$ de ellos conoce a su tutor y muestran satisfacción y confianza al contar con este apoyo por parte de la institución.

Con el sistema el tutor actualmente cuenta con información que le permite dar continuidad a la acción tutorial en los semestres siguientes, como se manifestó en las entrevistas. 
Por estas razones en el Instituto Tecnológico de Tehuacán, se cuenta con un sistema Integral que involucra a cada uno de los actores, que hasta el momento está favoreciendo al programa de tutorías, con esto se concluye que el objetivo del trabajo de investigación se cumple y la respuesta a la pregunta de investigación es que con la implementación del sistema que integra a todos los actores del programa de tutorías y automatiza sus actividades, si se favorece al programa.

\section{Sugerencias}

Se recomienda que la institución a través del departamento de Desarrollo Académico, apoye con la capacitación de tutores para que no solo se favorezca sino también se logre impactar en los índices de reprobación y deserción y con esto lograr el objetivo de la tutoría e incidir en las metas institucionales, lograr que el $100 \%$ de tutores cursen el diplomado en formación de tutores. También que se realicen reuniones para retroalimentar y fortalecer el programa de tutorías y el uso del sistema integral de tutorías.

Se han detectado como resultado de este trabajo áreas de oportunidad que deben ser atendidas para mejorar el desempeño académico de los alumnos y elevar los índices de aprobación.

Como se mencionó una vez que los diferentes actores estén familiarizados con el uso del sistema y que los tutores reciban capacitación continua para el desempeño de su función, se podrá realizar una investigación que permita evaluar el impacto que tiene el uso de un sistema web que automatiza las actividades de los diferentes actores, en los objetivos de las tutorías que son disminuir los índices de reprobación y deserción.

\section{Agradecimientos}

Se externa el agradecimiento a las siguientes instancias que hicieron posible la realización del proyecto: "Sistema Integral para la Gestión del Programa de Tutorías en el Instituto Tecnológico de Tehuacán".

Al Tecnológico Nacional de México por el financiamiento otorgado para el desarrollo del proyecto.
- A los directivos en especial a la directora del Instituto Tecnológico de Tehuacán C. M.E. Yeyetzín Sandoval González, por todo su apoyo y participación en el proyecto.

- $\quad$ A la M.P.H. Patricia Huerta Orozco jefa del departamento de Desarrollo Académico por las facilidades y el apoyo brindado para las diferentes actividades realizadas hasta la culminación del proyecto.

A los alumnos que participaron y colaboraron con la realización del proyecto:

Martín Cruz Medinilla

Fernando Nuevo Valerio

Alejandro Marroquín Márquez

Liliana Correa Barragán

\section{Referencias}

aerus. (27 de abril de 2016). Ventajas de los sistemas web. Obtenido de https://www.aeurus.cl/recomendaciones/2016/0 4/27/ventajas-de-los-sistemas-web/

Alvarez, M. A. (2 de Enero de 2014). Qué es $M V C$. Recuperado el 1 de Febrero de 2017, de DesarrolloWeb.com:

https://desarrolloweb.com/articulos/que-esmvc.html

ANUIES. (sf).

PROGRAMAS

INSTITUCIONALES DE TUTORIA. UNA PROPUESTA DE LA ANUIES PARA SU ORGANIZACIÓN Y FUNCIONAMIENTO EN LAS INSTITUCIONES DE EDUCCAIÓN SUPERIOR. Obtenido de http://evirtual.uaslp.mx/FCQ/tutorias/Document os\%20compartidos/INTRODUCCION/PROGR AMAS\%20INSTITUCIONALES\%20DE\%20T UTORIA_ANUIES.pdf

Barrios, M. V. (2014). UNA PROPUESTA DE EVALUACIÓN PARA EVALUAR LA EFICIENCIA DELA TUTORÍA EN LA EDUCACIÓN NORMAL. EL CASO DE LA ENSEM. Universidad Nacional autónoma de Mexico, 1-11. 
Colmenares E., A. M., \& Piñeiro M., M. L. (2008). La investigación acción. Una herramienta metodológica heurística para la comprensión y transformación de realidades y prácticas socio-educativas. Obtenido de http://www.redalyc.org/pdf/761/76111892006.p df

EcuRed. (s.f.). Servidor web. Obtenido de https://www.ecured.cu/Servidor_Web

García , O. I. (agosto de 2018). Análisis de las tutorías académicas y sus efectos en alumnos y tutores del Instituto Tecnológico de Tehuacán. Tehuacán: IESE.

Latorre, A. (2005). La investigación-acción. En A. Latorre. España: Graó.

Monje, Á. C. (2011). Metodología de la investigación cuantitativa y cualitativa. Obtenido de https://www.uv.mx/rmipe/files/2017/02/Guiadidactica-metodologia-de-la-investigacion.pdf

Ricoy Lorenzo, C. (2006). Contribución sobre los paradigmas de investigación. Obtenido de Sistema de Información Científica Redalyc: http://www.redalyc.org/comocitar.oa?id=11711 7257002

Rizzo, V. L., \& Córdova, A. L. (abril de 2015). Análisis, desarrollo e implementación de una aplicación web para la gestión y publicación de hojas de vida y portafolio de trabajp de profesionales freelance, utilizando extreme programming y tecnologías open source. Obtenido de https://dspace.ups.edu.ec/bitstream/123456789/ 10309/1/UPS-GT001208.pdf

Rodríguez, R. F., García, O. I., \& Vásquez, G. S. (2016). Sistema web: herramienta para la gestión del programa de tutorías. Revista electrónica ANFEI digital, 1-9.

TecNM. (Febrero de 2013). Obtenido de MANUAL DEL TUTOR DEL SNIT: http://www.tecnm.mx/images/areas/docencia01 /documentos/MANUAL_DEL_TUTOR.pdf
TecNM. (2013). Cuaderno de trabajo de tutoría del estudiante. Obtenido de https://www.tecnm.mx/images/areas/docencia/2 012-

1/cuaderno/CUADERNO_DE_TRABAJO_DE _TUTORIA_DEL_ESTUDIANTE.pdf

TecNM. (4 de noviembre de 2015). Actualiza TecNM formación de tutores. Obtenido de https://www.tecnm.mx/academicas/tec-deatitalaquia-al-nacional-de-conacyt-dp1

Tehuacán, I. T. (16 de 01 de 2016). Programa Institucional de Innovación y Desarrollo 2013 2018 Del Instituto Tecnológico de Tehuacán. Obtenido de http://www.ittehuacan.edu.mx/images/images/f eb2017/PIIDitt2013_2018.pdf

Torres, G. I. (2 de 04 de 2013). La utilización de las TICS en las tutorías como medio para mejorar las relaciones entre centrosprofesores-padres-alumnos. Obtenido de https://reunir.unir.net/bitstream/handle/1234567 89/1798/2013_04_04_TFM_ESTUDIO_DEL_ TRABAJO.pdf? sequence $=1$ 